\title{
Caracterização e desempenho reprodutivo de fêmeas suínas submetidas à intervenção obstétrica manual
}

\author{
Characterization and reproductive performance of swine females submitted to manual obstetric \\ intervention
}

\author{
Ana Paula Gonçalves Mellagi ${ }^{\mathrm{I}}$ Giseli Heim ${ }^{\mathrm{II}}$ Mari Lourdes Bernardi' ${ }^{\mathrm{II}}$ \\ Fernando Pandolfo Bortolozzo ${ }^{* \mathrm{IV}}$ Ivo Wentz ${ }^{\mathrm{IV}}$
}

\section{RESUMO}

Os objetivos do presente estudo foram determinar as características de partos submetidos à intervenção manual e avaliar os efeitos dessa intervenção no desempenho reprodutivo subsequente e na taxa de remoção. Dados de 4121 partos foram separados em grupos Controle (3271 partos) $e$ Intervenção (850 partos). O percentual de partos submetidos à intervenção manual foi de 20,6\%. Não houve diferença $(P>0,05)$ na ocorrência de intervenção ao parto de acordo com as classes de tamanho de leitegada $(<11,11-14$ e $>14$ leitões). A proporção de intervenção ao parto aumentou $(P<0,05)$ no verão e conforme o aumento da ordem de parto $(\mathrm{OP})$. Nos partos com intervenção, foi constatada maior duração do parto $(P<0,05)$, nas fêmeas $O P 1$, maior número de leitões natimortos e maior frequência de partos com pelo menos um natimorto $(P<0,05)$, nas fêmeas $O P 1$, OP 3-5 e OP 6-10. No grupo Intervenção, maior intervalo desmame-estro (IDE), menor taxa de parto e menor tamanho da leitegada $(P<0,05)$ foram observados nas fêmeas OP 1-2, OP 3-5 e OP 6-10, respectivamente. A taxa de remoção antes da inseminação no primeiro estro pós-desmame, tanto por causas reprodutivas, como não reprodutivas, foi maior $(P<0,05)$ no grupo Intervenção. A intervenção manual ao parto é efetuada com maior frequência no verão e em fêmeas mais velhas. A intervenção obstétrica manual compromete o IDE, a taxa de parto ou o tamanho da leitegada subsequente.

Palavras-chave: estação do ano, natimortalidade, parto, remoção de fêmeas, reprodução.

\begin{abstract}
The aim of this study was to determine the characteristics of farrowings with manual intervention and to evaluate the effect of this intervention on the subsequent reproductive performance and culling rate. Data of 4121 farrowings were separated in Control (3271 farrowings) and Intervention (850 farrowings) groups. The percentage of farrowings submitted to manual intervention was $20.6 \%$. There was no difference $(P>0.05)$ in the occurrence of intervention according to litter size classes ( $<11,11-14$ and $>14$ piglets). The proportion of intervention at parturition increased $(P<0.05)$ during summer and in higher parity order (PO) females. In farrowings with intervention there were longer farrowing duration in PO 1 females $(P<0.05)$ and greater number of stillborn piglets and higher frequency of farrowings with at least one stillborn $(P<0.05)$ in PO 1, PO 3-5 and PO 6-10 females. In the Intervention group, greater weaning to estrus interval (WEI), lower farrowing rate and lower subsequent total born piglets $(P<0.05)$ were observed in $P O 1$ and 2, PO 3-5 and $P O$ 6-10, respectively. Removal rate before first insemination post weaning, by both reproductive and non reproductive reasons, was greater $(P<0.05)$ in Intervention group. Manual intervention at parturition is more frequent during summer and among older females. Manual obstetric intervention compromise the WEI, the farrowing rate or the subsequent litter size.
\end{abstract}

Key words: parturition, removal of females, reproduction, season, stillbirth.

'Programa de Pós-graduação em Ciências Veterinárias (PPGCV), Universidade Federal do Rio Grande do Sul (UFRGS). Porto Alegre, RS, Brasil.

${ }^{\text {IIC } C u r s o ~ d e ~ M e d i c i n a ~ V e t e r i n a ́ r i a, ~ S e t o r ~ d e ~ S u i ́ n o s, ~ U F R G S, ~ P o r t o ~ A l e g r e, ~ R S, ~ B r a s i l . ~}$

IIIDepartamento de Zootecnia, Faculdade de Agronomia, UFRGS, Porto Alegre, RS, Brasil.

${ }^{\text {Iv }}$ Departamento de Medicina Animal, Faculdade de Veterinária, UFRGS. Av. Bento Gonçalves, 9090, 91540-000, Porto Alegre, RS, Brasil. E-mail: fpbortol@ufrgs.br. *Autor para correspondência. 


\section{INTRODUÇÃO}

Na suinocultura, o número de leitões nascidos vivos é um parâmetro importante de produtividade, devido à influência direta no número de leitões desmamados por fêmea/ano (DIAL et al., 1992). Para maximizar esse índice, deve-se melhorar o atendimento ao parto, já que este representa um momento crítico para obtenção de maior número de leitões nascidos vivos (KNOX, 2005).

As distocias em suínos não são comuns quando comparadas às outras espécies, ocorrendo em menos de 3\% dos partos (SMITH, 1997; BRITT et al., 1999). Os fatores relacionados à ocorrência de distocia, segundo JACKSON (1973) e BRITT et al. (1999), são inércia uterina, mau posicionamento do leitão no canal do parto, presença de mais de um feto no canal do parto, rotação uterina e fetos muito grandes. A distocia aumenta a incidência de leitões natimortos, principalmente por prolongar a duração do parto (JACKSON, 1975). BORGES et al. (2005) verificaram que a chance de ocorrência de natimortos é duas vezes maior nos partos com mais de três horas. Nos partos prolongados, há maior probabilidade de ocorrência da ruptura prematura do cordão umbilical, levando à hipóxia e, possivelmente, lesão cerebral irreversível nos leitões (KNOX, 2005).

A intervenção obstétrica manual é útil quando o intervalo entre os nascidos excede 20-30 minutos, para evitar a exaustão da fêmea (MEREDITH, 1995; KNOX, 2005), desde que outras atitudes já tenham sido tomadas, como estimular a fêmea a levantar e fazer massagem abdominal. Apesar de ser recomendada para diminuir a natimortalidade, a intervenção manual no parto pode aumentá-la, caso não seja realizada corretamente (LUCIA Jr et al., 2002). Entretanto, no trabalho de BORGES et al. (2005), a intervenção manual não foi considerada fator de risco para natimortalidade.

Deve ser considerado que a intervenção manual precoce e errônea pode promover distúrbios no parto natural (RUNNELS, 1980), ocasionando ferimentos do tecido do canal do parto (MEREDITH, 1995), morte dos fetos, diminuição da viabilidade dos leitões, infecções locais ou sistêmicas ou, até mesmo, morte da fêmea (BRITT et al., 1999). Apesar de necessária para ocasiões de distocia, a intervenção obstétrica manual é um método invasivo, e agentes com potencial patogênico podem ser introduzidos no útero, comprometendo o desempenho reprodutivo subsequente (RUNNELS, 1980).

Há pouca informação disponível sobre as consequências da intervenção obstétrica manual. Tendo em vista o aumento de produtividade das fêmeas modernas, em termos de número de leitões nascidos, e a provável necessidade de maior intervenção ao parto, é importante estudar o seu efeito no desempenho reprodutivo posterior. O presente estudo visou avaliar o efeito da ordem de parto, tamanho da leitegada e estação do ano sobre a ocorrência de intervenções manuais realizadas durante o parto e determinar as características produtivas das fêmeas suínas submetidas a essa intervenção, além de verificar as consequências dessa prática sobre as taxas de remoção das fêmeas e o desempenho reprodutivo subsequente.

\section{MATERIAL E MÉTODOS}

O estudo foi conduzido em uma Unidade Produtora de Leitões com 1.800 matrizes, da genética Agroceres PIC ${ }^{\circledR}$, na região da Serra Gaúcha (Latitude 2909'46.00” Sul e Longitude 5144'13.03” Oeste), no Rio Grande do Sul, no período de março de 2005 a abril de 2006. Foram coletados dados de fichas das fêmeas referentes a 4121 partos (data do parto, ordem de parto, número de leitões nascidos vivos, natimortos, mumificados e ocorrência de intervenção obstétrica manual), os quais foram separados em grupo Controle (3271 partos) e Intervenção (850 partos). A vacinação para doenças reprodutivas (parvovirose, leptospirose e erisipelose) foi realizada na maternidade, 15 dias antes da inseminação para as pluríparas; e 30 e 15 dias antes da primeira inseminação de leitoas.

A intervenção realizada pela introdução da mão e do braço no canal vaginal e/ou útero durante o trabalho de parto, definida como intervenção obstétrica manual, foi realizada após higiene do posterior das fêmeas e com o uso de luva descartável e gel. A decisão de prestar o auxílio foi baseada no intervalo entre nascidos (maior que 30 minutos) e na dificuldade em expulsar os leitões (deficiência nas contrações abdominais ou uterinas).

A ordem de parição (OP) foi dividida em OP 1, OP 2, OP 3-5 e OP 6-10. O tamanho da leitegada foi classificado em <11, 11-14 e >14 leitões. As estações foram classificadas em verão, outono, inverno e primavera. No período avaliado, as variações na média da temperatura ambiental diária (amplitude mínima e máxima), no verão, outono, inverno e primavera, foram de 16 a $26^{\circ} \mathrm{C}$ (amplitude de $11-33^{\circ} \mathrm{C}$ ), 6 a $26^{\circ} \mathrm{C}$ (amplitude de $3-31^{\circ} \mathrm{C}$ ), 4 a $21^{\circ} \mathrm{C}$ (amplitude de $3-29^{\circ} \mathrm{C}$ ), 9 a $26^{\circ} \mathrm{C}$ (amplitude de $5-31^{\circ} \mathrm{C}$ ), respectivamente (INMET, 2008).

A duração do parto foi considerada como sendo o intervalo entre as expulsões do primeiro e último leitão, em minutos. Foi analisado o desempenho reprodutivo subsequente nas fêmeas inseminadas no primeiro estro após o desmame correspondente à 
lactação dos partos avaliados. Também foi avaliada a remoção das fêmeas no ciclo produtivo subsequente ao parto avaliado. Os motivos de remoção foram obtidos a partir do banco de dados de programa de gerenciamento. Foram consideradas as remoções efetuadas antes ou após a inseminação artificial no primeiro estro após o desmame. As causas de remoção foram divididas em causas reprodutivas e não reprodutivas. Foi considerada, para a remoção por causas reprodutivas, a ocorrência de descargas vulvares advindas de infecção urinária e/ou uterina, anestro após o desmame, retorno, abortamento e fêmeas vazias ao parto. As causas não reprodutivas incluíram problemas locomotores, fêmeas em decúbito, doenças, morte e sacrifício.

Os percentuais de partos com intervenção, de acordo com as classes de OP, o tamanho da leitegada e a estação do ano, além da frequência de partos com pelo menos um natimorto, foram obtidos pelo procedimento FREQ (SAS, 1998), e a comparação foi realizada pelo teste do qui-quadrado. A taxa de parto foi calculada considerando todas as fêmeas inseminadas, ao passo que a taxa de parto ajustada foi calculada excluindo as fêmeas que não pariram por causas não reprodutivas. As taxas de retorno, de abortamento, de parto, de parto ajustada e a taxa de remoção foram comparadas pelo teste do qui-quadrado. Para análise do número de leitões nascidos totais, dos nascidos vivos e da duração do parto, foi utilizado o procedimento GLM (SAS, 1998), e as médias foram comparadas pelo teste $t$. O número total de leitões nascidos no parto avaliado foi utilizado como covariável na análise do total de leitões nascidos no parto subseqüente. Os natimortos e mumificados foram analisados pelo procedimento NPAR1WAY (SAS, 1998), e os grupos foram comparados pelo teste de Wilcoxon. Inseminações seguidas de retorno ou abortamento não foram incluídas na análise do tamanho da leitegada subsequente, visto que essa variável poderia não mais estar sob a influência da intervenção manual efetuada no parto anterior e sim do maior intervalo entre partos.

\section{RESULTADOS E DISCUSSÃO}

O percentual de partos submetidos à intervenção manual foi de 20,6\%, próximo de 22,8\% observado em estudo efetuado em quatro granjas brasileiras (BORGES et al., 2005). SOBESTIANSKY et al. (2001) consideraram que a taxa de auxílio obstétrico deve ficar em torno de $10 \%$, a fim de evitar intervenções desnecessárias. De fato, se forem considerados os relatos de cerca de $3 \%$ de distocia em suínos (RANDALL, 1972; RUNNELS \& CLARK, 1992), os percentuais de intervenção ao parto não deveriam ser tão elevados quanto os observados nesses estudos. É possível que, apesar das recomendações corretas, algumas intervenções possam ter sido efetuadas de forma indiscriminada, por excesso de cuidado, na tentativa de reduzir a ocorrência de natimortos intraparto, visto que estes representam de $51 \%$ a $77 \%$ do total de perdas por natimortalidade (SCHNEIDER, 2002). Além disso, as metas de percentuais de natimortalidade podem ser utilizadas para a avaliação dos funcionários de maternidade, o que pode levá-los ao aumento de intervenção no parto para tentar diminuir o número de natimortos e aumentar o número de leitões nascidos vivos. Assim, mesmo que a intervenção possa contribuir para a redução da natimortalidade, é importante que os funcionários estejam conscientes de que a intervenção é um método invasivo e pode comprometer o desempenho subsequente das fêmeas.

Os percentuais de intervenção obstétrica, de acordo com o tamanho de leitegada, foram de 20,5\%, $20,2 \%$ e 21,6\% para as classes $<11$ leitões, 11 a 14 leitões e $>14$ leitões, respectivamente, não tendo sido observada diferença significativa $(\mathrm{P}>0,05)$. BORGES et al. (2005), entretanto, relataram índices de intervenção diferentes de acordo com a classe de tamanho da leitegada, pois as fêmeas com menos de 10 leitões tiveram 32\% de intervenção manual, superior aos $24 \%$ e $18 \%$ para as fêmeas com 10-12 e >12 leitões, respectivamente. Segundo os autores, em leitegadas com poucos leitões, a intervenção manual nem sempre teria sido conduzida para minimizar o efeito de uma distocia, mas para certificar que não havia mais leitões no canal do parto e, assim, o funcionário poderia registrar o parto como encerrado.

As médias de OP não diferiram estatisticamente entre as estações $(\mathrm{P}>0,05)$, sendo de 3,8 (outono), 4,0 (inverno), 3,8 (primavera) e 3,7 (verão) partos. Na tabela 1, é apresentada a distribuição da ocorrência de intervenção obstétrica conforme as classes de ordem de parto e as estações do ano. Em todas as classes de ordem de parto, maior percentual de intervenção $(\mathrm{P}<0,05)$ ocorreu no verão. Houve maior percentual $(\mathrm{P}<0,05)$ de intervenção manual nos partos de fêmeas mais velhas (OP 6-10) do que nas demais classes, em todas as estações do ano. A dificuldade no trabalho de parto pode ser atribuída ao maior escore corporal visual, ao menor tônus muscular ou aos partos mais prolongados (PEJASK, 1984) em fêmeas mais velhas, contribuindo para a sua exaustão física, principalmente em situações de estresse térmico. A exaustão pode dificultar o trabalho de parto, pela redução das contrações abdominais e uterinas, o que explicaria a maior ocorrência de intervenção manual 
Tabela 1 - Frequência de distribuição da intervenção obstétrica ao parto de acordo com a ordem de parto e estação do ano

\begin{tabular}{|c|c|c|c|c|c|}
\hline Ordem de parto & outono & inverno & primavera & verão & total \\
\hline 1 & $\begin{array}{l}12 / 191 \\
(6,3) a A\end{array}$ & $\begin{array}{c}8 / 170 \\
(4,7) \mathrm{aA}\end{array}$ & $\begin{array}{l}14 / 281 \\
(5,0) \mathrm{aA}\end{array}$ & $\begin{array}{c}86 / 183 \\
(47,0) a B\end{array}$ & $\begin{array}{c}120 / 825 \\
(14,6) a\end{array}$ \\
\hline 2 & $\begin{array}{l}13 / 200 \\
(6,5) \mathrm{aA}\end{array}$ & $\begin{array}{c}6 / 174 \\
(3,4) \mathrm{aA}\end{array}$ & $\begin{array}{c}12 / 160 \\
(7,5) a b A\end{array}$ & $\begin{array}{c}93 / 223 \\
(41,7) a B\end{array}$ & $\begin{array}{l}124 / 757 \\
(16,4) a b\end{array}$ \\
\hline $3-5$ & $\begin{array}{l}18 / 288 \\
(6,2) a A\end{array}$ & $\begin{array}{l}24 / 413 \\
(5,8) \mathrm{aA}\end{array}$ & $\begin{array}{c}45 / 425 \\
(10,6) b B\end{array}$ & $\begin{array}{c}195 / 436 \\
(44,7) a C\end{array}$ & $\begin{array}{c}282 / 1562 \\
(18,1) b\end{array}$ \\
\hline $6-10$ & $\begin{array}{c}57 / 244 \\
(23,4) \mathrm{bA}\end{array}$ & $\begin{array}{c}42 / 259 \\
(16,2) b B\end{array}$ & $\begin{array}{c}87 / 246 \\
(35,4) c C\end{array}$ & $\begin{array}{c}138 / 228 \\
(60,5) b D\end{array}$ & $\begin{array}{l}324 / 977 \\
(33,2) c\end{array}$ \\
\hline Total & $\begin{array}{l}100 / 923 \\
(10,8) A\end{array}$ & $\begin{array}{c}80 / 1016 \\
(7,9) B\end{array}$ & $\begin{array}{c}158 / 1112 \\
(14,2) C\end{array}$ & $\begin{array}{c}512 / 1070 \\
(47,8) \mathrm{D}\end{array}$ & -- \\
\hline
\end{tabular}

abc na coluna indicam diferença significativa entre as ordens de parto $(\mathrm{P}<0,05)$.

$A B C D$ na linha indicam diferença significativa entre as estações $(P<0,05)$.

nos períodos mais quentes do ano. Cabe salientar que o verão de 2006 foi chuvoso, com número elevado de dias com umidade do ar próxima ou acima de $80 \%$ (INMET, 2008). Assim, a associação de altas temperaturas com alta umidade pode ter contribuído para maior dificuldade de parto e exaustão das fêmeas.

Nas classes OP 1, OP 3-5 e OP 6-10, os partos com intervenção estiveram associados ao maior número de leitões natimortos (Tabela 2) e maior percentual de partos com pelo menos um natimorto $(\mathrm{P}<0,05)$. Maiores OP estão geralmente associadas a maiores chances de natimortalidade (RANDALL \& PENNY, 1970; LUCIA Jr et al., 2002; SCHNEIDER, 2002; BORGES et al., 2005), o que pode estar relacionado à maior gordura corporal de fêmeas mais velhas (MUIRHEAD \& ALEXANDER, 2001) ou ao aumento na duração do parto (CAVALCANTI et al., 1979). SCHNEIDER et al. (2001) observaram $12,2 \%$ de natimortos em fêmeas $\mathrm{OP}>5$, valor acima de 7\%, que é o máximo aceitável (DIAL et al., 1992). Em outro estudo, $51 \%$ das fêmeas OP $>5$ tiveram natimortos (BORGES et al., 2005). No presente estudo, 35\% das fêmeas OP 6-10 tiveram natimortos e, mesmo naquelas que sofreram intervenção, o percentual de natimortos não ultrapassou 5\% do total de leitões nascidos, o que sugere que a intervenção efetuada nessas fêmeas evitou as elevadas taxas de natimortalidade citadas anteriormente. Por outro lado, o percentual de partos com natimortos nas fêmeas OP 1 foi 32\%, próximo de 33\% observado por BORGES et al. (2005). Nos partos com intervenção, houve 5,4\% de natimortos nas OP 1, semelhante a 5,0\% observado nas fêmeas OP 6-10, sugerindo que a intervenção foi menos eficiente nas OP 1 . É possível que os funcionários tenham prestado assistência mais adequada às fêmeas mais velhas, já que há expectativa de maior ocorrência de problemas de parto e maior natimortalidade nessa categoria.

Maior ocorrência de natimortos está associada a partos mais prolongados, e os leitões natimortos são expulsos em intervalos mais longos do que os observados nos leitões vivos (FRASER et al., 1997; VAN DIJK et al., 2005). No entanto, não está claro se os natimortos são a causa ou o resultado de um parto prolongado, pois partos prolongados aumentam a probabilidade de asfixia e morte, mas, se o leitão já está morto, ele é incapaz de realizar os movimentos necessários para alcançar o canal do parto e para desencadear as contrações uterinas necessárias para sua expulsão (VAN DIJK et al., 2005). A intervenção obstétrica é normalmente efetuada com o objetivo de facilitar o trabalho de parto e reduzir as perdas por natimortalidade do tipo intraparto. Como o tipo de natimortalidade não foi determinado, no presente estudo, algumas intervenções podem ter acontecido pela existência de natimortos do tipo pré-parto. Nesses casos, a intervenção poderia evitar o prolongamento do parto, mas a natimortalidade do tipo pré-parto não teria sido evitada. Por outro lado, é difícil saber se perdas por natimortalidade intraparto ocorreriam ou não, caso a intervenção não fosse efetuada.

Houve comprometimento do desempenho reprodutivo subsequente, o qual foi constatado por menor IDE nas fêmeas OP 1 e OP 2, menor taxa de parto nas fêmeas OP 3-5 e menor tamanho da leitegada nas 
Tabela 2 - Características de partos de acordo com a realização ou não de intervenção manual obstétrica em diferentes classes de ordem de parto (OP)

\begin{tabular}{|c|c|c|c|c|c|c|c|c|}
\hline OP & Grupo & DPART (min) & NTOT & NVIV & NATI & \% de NATI & PFNATI & MUMI \\
\hline \multirow{3}{*}{1} & $\begin{array}{c}\text { Controle } \\
n=705\end{array}$ & $207 \pm 104$ & $12,1 \pm 3,0$ & $11,5 \pm 3,0$ & $0,38 \pm 0,63$ & $3,0 \pm 5,3$ & 30,9 & $0,30 \pm 0,67$ \\
\hline & $\begin{array}{c}\text { Intervenção } \\
n=120\end{array}$ & $243 \pm 144$ & $12,2 \pm 3,0$ & $11,1 \pm 3,3$ & $0,63 \pm 1,0$ & $5,4 \pm 8,3$ & 40,8 & $0,43 \pm 0,91$ \\
\hline & $\mathrm{P}$ & 0,002 & 0,91 & 0,23 & 0,007 & 0,003 & 0,032 & 0,23 \\
\hline \multirow{3}{*}{2} & $\begin{array}{c}\text { Controle } \\
n=633\end{array}$ & $206 \pm 107$ & $11,9 \pm 2,9$ & $11,4 \pm 2,9$ & $0,29 \pm 0,58$ & $2,3 \pm 5,1$ & 23,2 & $0,20 \pm 0,56$ \\
\hline & $\begin{array}{c}\text { Intervenção } \\
n=124\end{array}$ & $220 \pm 133$ & $11,5 \pm 3,3$ & $10,8 \pm 3,2$ & $0,44 \pm 0,92$ & $3,8 \pm 7,9$ & 28,2 & $0,29 \pm 0,67$ \\
\hline & $\mathrm{P}$ & 0,30 & 0,26 & 0,05 & 0,16 & 0,119 & 0,233 & 0,16 \\
\hline \multirow{3}{*}{$3-5$} & $\begin{array}{l}\text { Controle } \\
n=1280\end{array}$ & $215 \pm 115$ & $12,9 \pm 3,0$ & $12,3 \pm 2,9$ & $0,34 \pm 0,63$ & $2,6 \pm 5,0$ & 28,9 & $0,26 \pm 0,58$ \\
\hline & $\begin{array}{c}\text { Intervenção } \\
n=282\end{array}$ & $232 \pm 129$ & $12,8 \pm 3,5$ & $11,9 \pm 3,4$ & $0,46 \pm 0,67$ & $3,6 \pm 5,6$ & 36,9 & $0,37 \pm 0,69$ \\
\hline & $\mathrm{P}$ & 0,06 & 0,59 & 0,09 & 0,005 & 0,002 & 0,008 & 0,01 \\
\hline \multirow{3}{*}{$6-10$} & $\begin{array}{c}\text { Controle } \\
n=653\end{array}$ & $198 \pm 111$ & $12,7 \pm 3,0$ & $12,1 \pm 2,8$ & $0,36 \pm 0,59$ & $2,8 \pm 4,7$ & 30,6 & $0,24 \pm 0,53$ \\
\hline & $\begin{array}{c}\text { Intervenção } \\
n=324\end{array}$ & $210 \pm 148$ & $12,7 \pm 3,3$ & $11,8 \pm 3,1$ & $0,65 \pm 0,95$ & $5,0 \pm 7,3$ & 43,8 & $0,21 \pm 0,53$ \\
\hline & $\mathrm{P}$ & 0,23 & 0,87 & 0,14 & $<0,0001$ & $<0,0001$ & $<0,0001$ & 0,31 \\
\hline
\end{tabular}

$\mathrm{OP}=$ ordem de parto; DPART= duração do parto (fase de expulsão dos leitões); NTOT= total de leitões nascidos; NVIV= leitões nascidos vivos; NATI= natimortos; MUMI= mumificados; PFNATI= percentual de fêmeas com natimortos. P indica o valor de probabilidade observado na análise estatística para cada variável.

fêmeas OP 6-10 (Tabela 3). Segundo RUNNELS (1980), ao levar patógenos para o ambiente uterino, a intervenção obstétrica manual pode comprometer a próxima gestação, tanto na sua manutenção, quanto no número de leitões produzidos. LEBRET (1999) considerou que a intervenção manual, no parto anterior, é um dos fatores de risco para leitegadas pequenas ( $=8$ leitões), pois, das fêmeas com leitegadas pequenas, $26 \%$ haviam sido submetidas à intervenção manual no parto anterior, enquanto que, nas fêmeas com mais de 8 leitões, o índice de intervenção era de $16 \%$.

A taxa geral de remoção foi maior $(\mathrm{P}<0,0001)$ para o grupo Intervenção (Tabela 4). As remoções desse grupo se caracterizaram por serem de maior OP, menor número de nascidos vivos e maior número de natimortos, quando comparadas às remoções do grupo Controle. A remoção foi maior no grupo Intervenção, antes da inseminação no primeiro estro após o desmame, tanto por problemas reprodutivos, quanto por não reprodutivos. As causas reprodutivas de remoção antes da inseminação foram anestro após o desmame e secreções vulvares, o que pode ser consequência da intervenção ao parto. O maior descarte por causas não reprodutivas pode ser atribuído à idade das fêmeas, uma vez que apresentaram maior média de partos $(P<0,001)$. Numa compilação de 19 trabalhos, nos quais foram abordadas as causas de remoção, os principais motivos de remoção foram falha reprodutiva, baixa performance no parto, idade avançada e problemas no sistema locomotor (STALDER et al., 2004). Alêm disso, segundo esses autores, as falhas reprodutivas e os problemas locomotores são causas mais comuns em fêmeas com menos de três partos, ao passo que fêmeas mais velhas são removidas principalmente por baixa performance no parto, idade e até mesmo morte. A maior taxa de descarte por causas não reprodutivas, observada no presente estudo, não está necessariamente associada ao fato de as fêmeas terem sido submetidas à intervenção manual durante o parto, mas ao fato de serem mais velhas, o que implicaria em maior propensão a problemas locomotores ou ao desgaste corporal por sucessivas lactações, justificando sua maior taxa de descarte.

\section{CONCLUSÃO}

De acordo com as condições em que o trabalho foi realizado, conclui-se que a intervenção

Ciência Rural, v.39, n.5, ago, 2009. 
Tabela 3 - Desempenho reprodutivo subsequente de acordo com a realização ou não de intervenção manual obstétrica em diferent es classes de ordem de parto (OP)

\begin{tabular}{|c|c|c|c|c|c|c|c|}
\hline OP & Grupo & IDE & TRET & TABR & $\mathrm{TP}$ & TPA & NTOT \\
\hline \multirow[t]{3}{*}{1} & $\begin{array}{c}\text { Controle } \\
n=679\end{array}$ & $5,7 \pm 4,8$ & $37(5,4)$ & $9(1,3)$ & $624(91,9)$ & $\begin{array}{c}624 / 674 \\
(92,6)\end{array}$ & $11,9 \pm 3,0$ \\
\hline & $\begin{array}{c}\text { Intervenção } \\
n=104\end{array}$ & $8,0 \pm 8,1$ & $6(5,8)$ & $1(0,96)$ & $95(91,3)$ & $\begin{array}{c}95 / 102 \\
(93,1)\end{array}$ & $11,9 \pm 2,9$ \\
\hline & $\mathrm{P}$ & $<0,0001$ & 0,894 & 1,00 & 0,848 & 0,841 & 0,834 \\
\hline \multirow[t]{3}{*}{2} & $\begin{array}{c}\text { Controle } \\
n=608\end{array}$ & $4,5 \pm 3,3$ & $29(4,8)$ & $4(0,7)$ & $562(92,4)$ & $\begin{array}{c}562 / 596 \\
(94,3)\end{array}$ & $12,8 \pm 3,1$ \\
\hline & $\begin{array}{c}\text { Intervenção } \\
n=110\end{array}$ & $5,2 \pm 4,2$ & $6(5,4)$ & $2(1,8)$ & $102(92,7)$ & $\begin{array}{c}102 / 110 \\
(92,7)\end{array}$ & $12,4 \pm 3,2$ \\
\hline & $\mathrm{P}$ & 0,05 & 0,759 & 0,231 & 0,915 & 0,523 & 0,226 \\
\hline \multirow[t]{3}{*}{$3-5$} & $\begin{array}{l}\text { Controle } \\
n=1172\end{array}$ & $5,0 \pm 4,7$ & $67(5,7)$ & $23(2,0)$ & $1055(90,0)$ & $\begin{array}{c}1055 / 1150 \\
(91,7)\end{array}$ & $12,8 \pm 3,3$ \\
\hline & $\begin{array}{c}\text { Intervenção } \\
n=232\end{array}$ & $5,2 \pm 4,3$ & $20(8,6)$ & $10(4,3)$ & $194(83,6)$ & $\begin{array}{c}194 / 225 \\
(86,2)\end{array}$ & $12,7 \pm 3,2$ \\
\hline & $\mathrm{P}$ & 0,54 & 0,094 & 0,031 & 0,0045 & 0,0087 & 0,789 \\
\hline \multirow[t]{3}{*}{$6-10$} & $\begin{array}{c}\text { Controle } \\
n=516\end{array}$ & $4,8 \pm 4,4$ & $26(5,0)$ & $11(2,1)$ & $462(89,5)$ & $\begin{array}{c}462 / 505 \\
(91,5)\end{array}$ & $12,5 \pm 3,2$ \\
\hline & $\begin{array}{c}\text { Intervenção } \\
n=218\end{array}$ & $4,7 \pm 4,0$ & $15(6,9)$ & $6(2,7)$ & $189(86,7)$ & $\begin{array}{c}189 / 212 \\
(89,1)\end{array}$ & $11,8 \pm 3,3$ \\
\hline & $\mathrm{P}$ & 0,78 & 0,321 & 0,610 & 0,267 & 0,324 & 0,018 \\
\hline
\end{tabular}

$\mathrm{OP}=$ ordem de parto; $\mathrm{n}=$ corresponde ao número de fêmea s inseminadas; IDE= intervalo desmame-estro; TRET= taxa de retorno; TABR= taxa de abortamento; $\mathrm{TP}=$ taxa de parto; $\mathrm{TPA}=$ taxa de parto ajustada (excluindo fêmeas que não pariram por causas não reprodutivas); NTOT = total de leitões nascidos.

$\mathrm{P}$ indica o valor de probabilidade observado na análise estatística para cada variável.

Tabela 4 - Taxas de remoção por causas reprodutivas e não reprodutivas em partos submetidos ou não à intervenção manual.

\begin{tabular}{|c|c|c|c|}
\hline & $\begin{array}{l}\text { Controle } \\
(\mathrm{n}=3271)\end{array}$ & $\begin{array}{c}\text { Intervenção } \\
(\mathrm{n}=850)\end{array}$ & $\mathrm{P}$ \\
\hline Total de remoções, \% (n) & $13,5(442)$ & $26,0(221)$ & $<0,0001$ \\
\hline Ordem de parto, $\mathrm{n}^{\circ} \pm \mathrm{dp}$ & $4,9 \pm 2,7$ & $5,7 \pm 2,8$ & 0,0006 \\
\hline Nascidos totais, $n^{\circ} \pm d p$ & $11,1 \pm 3,1$ & $10,9 \pm 3,7$ & 0,515 \\
\hline Nascidos vivos, $n^{\circ} \pm d p$ & $10,5 \pm 3,0$ & $10,0 \pm 3,6$ & 0,029 \\
\hline Natimortos, $\mathrm{n}^{\circ} \pm \mathrm{dp}$ & $0,33 \pm 0,70$ & $0,67 \pm 1,0$ & $<0,0001$ \\
\hline Mumi ficados, $n^{\circ} \pm d p$ & $0,25 \pm 0,53$ & $0,31 \pm 0,70$ & 0,828 \\
\hline Remoção por causas reprodutivas, \% (n) & $3,7(121)$ & 3,9 (33) & 0,802 \\
\hline Remoção por causas não reprodutivas, \% (n) & $9,8(321)$ & $22,1(188)$ & $<0,0001$ \\
\hline \multicolumn{4}{|c|}{ Remoção entre o parto e a inseminação pós-desmame } \\
\hline Total de remoções, \% (n) & $9,0(296)$ & $21,9(186)$ & $<0,0001$ \\
\hline Remoção por causas reprodutivas, \% (n) & $0,8(25)$ & $1,5(13)$ & 0,038 \\
\hline Remoção por causas não reprodutivas, \% (n) & $8,3(271)$ & $20,3(173)$ & $<0,0001$ \\
\hline \multicolumn{4}{|l|}{ Remoção após a inseminação } \\
\hline Total de inseminações & 2975 & 664 & \\
\hline Total de remoções, \% (n) & $4,9(146)$ & $5,3(35)$ & 0,697 \\
\hline Remoção por causas reprodutivas, \% (n) & 3,2 (96) & $3,0(20)$ & 0,776 \\
\hline Remoção por causas não reprodutivas, \% (n) & $1,7(50)$ & $2,3(15)$ & 0,309 \\
\hline
\end{tabular}


manual ao parto é efetuada com maior frequência no verão e em fêmeas mais velhas. Nos partos submetidos à intervenção obstétrica manual, o número de natimortos é maior. A intervenção obstétrica afeta o intervalo desmame-estro, a taxa de parto ou o tamanho da leitegada subsequente. A taxa de remoção antes da inseminação, no primeiro estro após o desmame, é maior nas fêmeas com intervenção manual no parto.

\section{REFERÊNCIAS}

BORGES, V.F. et al. Risk factors for stillbirth and foetal mummification in four Brazilian swine herds. Preventive Veterinary Medicine, v.70, p.165-176, 2005.

BRITT, J.H. et al. Diseases of the reproductive system. In: STRAW, B.E. et al. Diseases of swine. 8.ed. London: Iowa State University, 1999. Cap.60, p.883-911.

CAVALCANTI, S.S. et al. Efeito da duração do parto na incidência de leitões natimortos. Arquivos da Escola de Veterinária da UFMG, v.3, n.1, p.9-43, 1979.

DIAL, G.D. et al. Reproductive failure: differential diagnosis. In: LEMAN, A.D. et al. Diseases of swine. 7.ed. London: Iowa State University, 1992. Cap.6, p.88-137.

FRASER, D. et al. Farrowing behaviour and stillbirth in two environments: an evaluation of the restraint-stillbirth hypothesis. Applied Animal Behaviour Science, v.55, p.5166, 1997.

INMET, 2008. Gráficos das condições meteorológicas registradas. Capturado em 11 mai. 2008. Online. Disponível na Internet: http://www.inmet.gov.br/html/observacoes.php.

JACKSON, P.G.G. Causes of dystokia in the sow. Veterinary Record, v.93, n.3, p.77-78, 1973.

JACKSON, P.G.G. The incidence of stillbirth in cases of dystocia in sows. Veterinary Record, v.97, n.21, p.411-412, 1975.

KNOX, R.V. I mproving Farrowing Management. Capturado em 27 jul. 2005. Online. Disponível em: http:// porkinfo.osu.edu/Sow Mgm t\%20 ShortCourse/ Sow Mgmt\%20CD\%20Info'/Sow MgmtPDF12.02/ Improving\%20Farrowing\%20Management.pdf.

LEBRET, A. La gestion des petites portées. Porc Magazine, n.328, p.76-78, 1999.

LUCIA Jr, T. et al. Risk factors for stillbirths in two swine farms in the south of Brazil. Preventive Veterinary Medicine, v.53, p.285-292, 2002.

MEREDITH, M.J. Pig breeding and infertility. In: MEREDITH, M.J. Animal Breeding and Infertility. London: Blackwell Science,1995. Cap.7. p.278-353.
MUIRHEAD, M.R.; ALEXANDER, T.J.L. In: __ Manejo sanitario y tratamiento de las enfermedades del cerdo. Buenos Aires: InterMédica, 2001. Cap.8. p.263-322.

PEJASK, Z. Some pharmacological methods to reduce intrapartum death of piglets. Pig News and Information, v.5, p.35-37, 1984.

RANDALL, G.C.B.; PENNY, H.C. Stillbirth in the pig: an analysis of the breeding records of five herds. British Veterinary Journal, v.126, p.593-603, 1970.

RANDALL, G.C.B. Observations on parturition in the sow. Veterinary Record, v. 90, p.178-182, 1972.

RUNNELS, L.J. Obstetrics and cesarean section in swine. In: MORROW, D.A. Current therapy in theriogenology: diagnosis, treatment and prevention of reproductive diseases in animals. Philadelphia: Saunders, 1980. p.10681071.

RUNNELS, L.J.; CLARK, L.K. Obstetrics. In: LEMAN, A.D. et al. (Eds). Diseases of swine, 7.ed. Ames: Iowa State University, 1992. Cap.75, p.925-932.

SAS Institute INC. SAS user's guide: statistics. Cary, North Carolina, 1998.

SCHNEIDER, I.G. Natimortalidade suína em granjas industriais: distribuição, qualidade dos registros do parto e causas associadas à natimortalidade pré-parto, intraparto e pós-nascimento. 2002. 96f. Dissertação (Mestrado em Ciências Veterinárias) Faculdade de Veterinária, Universidade Federal do Rio Grande do Sul, Porto Alegre.

SCHNEIDER, I.G. et al. Avaliação da mumificação fetal e natimortalidade de acordo com o tamanho da leitegada e a ordem de parto. In: CONGRESSO BRASILEIRO DE VETERINÁRIOS ESPECIALISTAS EM SUÍNOS, 10., 2001, Porto Alegre, RS. Anais... Concórdia : Embrapa Suínos e Aves, 2001. V.2. 384p. p.203-204

SMITH, C.A. Normal and abnormal parturition in swine. In: YOUNGQUIST, R.S. Current therapy in large animal theriogenology. Philadephia: Saunders, 1997. Cap.106, p.719-726.

SOBESTIANSKY, J. et al. Visita ao sistema de produção. In: SOBESTIANSKY, J; BARCELLOS, D.E. Clínica veterinária em sistemas intensivos de produção de suínos e relatos de casos clínicos. Goiânia: Art 3, 2001. Cap.8. p.28-58.

Stalder, K.J. et al. Sow longevity. Pig News and Information, v.25, n.2, p.53-74, 2004.

VAN DIJK, A.J. et al. Factors affecting duration of the expulsive stage of parturition and piglet birth intervals in sows with uncomplicated, spontaneous farrowings. Theriogenology, v.64, p.1573-1590, 2005. 